\title{
Synchrotron FTIR characterisation of automotive primer surfacer paint coatings for forensic purposes
}

\author{
Mark Maric ${ }^{1}$, Wilhelm van Bronswijk ${ }^{1}$, Simon W. Lewis ${ }^{1 *}$, Kari Pitts $^{2}$ \\ ${ }^{1}$ Department of Chemistry, Curtin University of Technology, GPO Box U1987, Perth, \\ Western Australia 6845, Australia; \\ ${ }^{2}$ Chem Centre, Bentley Delivery Centre, PO Box 1250, Western Australia 6983, Australia; \\ *Author for correspondence: Simon W. Lewis \\ Department of Chemistry, Curtin University \\ GPO Box U1987 Perth, Western Australia 6845 \\ E-mail: S.Lewis@curtin.edu.au Tel: +61 (08) 92662484
}

\begin{abstract}
Synchrotron FTIR microspectroscopy was used to characterise and assess the chemical diversity of electrocoat primer, primer surfacer and basecoats of automotive paint samples from 75 vehicles representing a range of international car manufacturers. Significant diversity was found in the synchrotron FTIR data from the primer surfacer coats. Classification using principal component analysis revealed 14 discrete groups, which could be associated with the country of manufacture, the specific manufacturer and manufacturing plant, the year of manufacture of the vehicle and in some instances the number of layers in the paint system. The model generated from the primer surfacer was significantly more discriminating than a previous model generated from FTIR analysis of clear coats of the same vehicles. Analysis of the primer surfacer also avoids issues of possible environmental degradation and component migration seen with FTIR analysis of automotive clear coats. Providing such information concerning vehicle origin would aid in the development of investigative leads from the analysis of questioned automotive paint samples located at the scene of a crime or on the body/clothing of a hit and run victim.
\end{abstract}

Keywords: primer surfacer; automotive paint; synchrotron FTIR; trace evidence; forensic science 


\section{Introduction}

Automotive paint is arguably one of the most frequently encountered forms of trace evidence in automobile collisions, hit and run accidents or generally any incident involving a vehicle.[1, 2] In many instances, automotive paint is the most significant form of trace contact evidence encountered at the incident scene, thereby adding increased importance to its subsequent analysis.[3, 4] The Scientific Working Group for Materials Analysis (SWGMAT) have developed guidelines for the forensic analysis of paint, which is conducted via a suite of instrumental techniques that characterise both physical and chemical properties of the sample.[5] Although these analytical protocols have long been instituted, they largely rely on the forensic examiner's subjective interpretation of results, consequently raising serious concerns regarding human error and human observer bias. As a result, there is a requirement to establish meticulous statistical and scientific approaches to the interpretation of forensic evidence.

Chemometrics eliminates the subjectivity associated with the visual comparison of complex spectra from questioned and known samples, as it ensures that quantitative, impartial measures of the data are obtained.[6, 7] This is immensely significant considering forensic science is a discipline centred on the notion of forming objective conclusions based upon the analysis of physical evidence. In essence, chemometrics provides a statistical measure of how similar or dissimilar samples are thus improving the reproducibility, reliability and discrimination of the data, whilst simultaneously addressing issues such as human error and bias.[7-9]

Fourier transform infrared (FTIR) spectroscopy is widely recognised as the best nondestructive method available for the analysis of paint, as it facilitates rapid identification of the binder, resin and additive components in the sample.[4, 10] Extensive research into the application of FTIR spectroscopy to the forensic analysis of automotive paint has previously been reported.[11-14] We have recently shown that it is possible using attenuated total reflectance (ATR)-FTIR spectroscopy in combination with chemometrics to classify automotive clear coats from new or near-new Australian manufactured and imported vehicles into 9 classes which reflect the country of manufacture and in some instances the manufacturing plant.[15] Post market clear coat resprays were readily identified by their infrared spectra, without requiring chemometrics, and excluded from the principal component/linear discriminant analysis model. Moderate weathering of the clear coats by 
environmental exposure did not affect the outcomes from the model but significant weathering of the clear coat surface (10 or more years of environmental exposure) lead to samples being clearly identified as outliers.[16]

Whilst ATR-FTIR spectroscopy ensures that spectra derive from only the first few microns of the clear coat, this is not the case when the paint layers are separated physically by scraping and the scraping subsequently analysed by transmission IR spectroscopy and care must be taken when interpreting such data. In these situations IR chemical imaging provides an alternative as it enables the simultaneous acquisition of chemical and spatial information. Previous research has shown that focal plane array (FPA) detectors may be employed in the stratigraphic imaging of automotive paint cross-sections.[17] However, FPA based IR imaging using a conventional globar source is hindered by limited spatial resolution.[18] The spatial resolution in FTIR imaging can be drastically improved by utilising a synchrotron source as opposed to a conventional infrared globar source. Synchrotron sourced infrared light is in the order of 100-1000 times brighter than globar sources, which translates into a roughly 20-40 fold improvement in the signal to noise ratio at diffraction limited spatial resolutions. As the synchrotron infrared source is highly collimated the spatial resolution limit can be improved from $25 \mu \mathrm{m}$ (globar) down to $3 \mu \mathrm{m}$ (synchrotron). This is of particular significance in the infrared chemical imaging of thin multicomponent systems such as automotive paint, where high spatial resolution and therefore smaller apertures is a necessity.[18] A recent study conducted by the authors utilised synchrotron FTIR microspectroscopy to map the distribution of chemical components across automotive paint cross-sections, so as to assess the extent of interlayer component migration.[19] High spatial resolution synchrotron source transmission IR spectra of paint thin sections showed that migration of components between the various layers can and does occur. Melamine in particular appears to migrate between layers and its migration into the clear coat would lead to an incorrect classification/identification if transmission spectra of scraping were used in an ATR based model.[19]

In those instances where IR spectra of clear coats are inconclusive further information can be obtained from underlying automotive paint layers such as the primer surfacer coat. To the best of our knowledge there is no published research available in the open literature examining the chemical variability in the composition of the underlying automotive paint layers for forensic purposes. Consequently, the main aim of this study was to employ synchrotron FTIR microspectroscopy in combination with multivariate statistics to assess the 
diversity in a statistically large population of automotive electrocoat primer, primer surfacer and basecoat layers, with a view to identifying relationships between the chemical composition of the coating and the potential make, model and year of manufacture of the vehicle. 


\section{Materials and Methods}

\section{Sample Preparation}

Automotive paint samples (Prestige Sunroofs WA) were obtained from roof panels removed during the course of sunroof installation as previously described.[15, 16, 19] For each sample the make, model, year and vehicle identification number (VIN) was recorded. Transverse sections of the paint chips ( $8 \mu \mathrm{m}$ thick) were prepared by microtoming paint chips sandwiched between two rigid pieces of plastic.[19] After separation from the plastic, the microtomed sections were pressed flat between two micro-diamond cell windows (Thermo Scientific). As the samples remained flat the top window was removed prior to spectra acquisition.

\section{Sample Analysis}

All experiments were undertaken on the IR microspectroscopy (IRM) beamline of the Australian Synchrotron, Melbourne, Australia as previously described.[19] Briefly, the beamline consists of a conventional Bruker Vertex V80v FTIR spectrometer equipped with a liquid nitrogen cooled narrow-band mercury cadmium telluride (MCT) detector in conjunction with a Bruker Hyperion 2000 microscope (Bruker Optik GmbH, Ettlingen, Germany) with a motorised sample stage contained within an atmospheric purge box. FTIR spectroscopy of the cross sectioned samples was performed in transmission mode utilising an $\mathrm{X}$-Y step size of $2.5 \mu \mathrm{m}$, with a $5 \mu \mathrm{m}$ x $5 \mu \mathrm{m}$ sampling aperture. All spectra were acquired over the range of $3900-730 \mathrm{~cm}^{-1}$ at a spectral resolution of $4 \mathrm{~cm}^{-1}$ with 64 accumulated scans. Data acquisition was performed via Bruker Opus software (version 6.5).

\section{Data Analysis}

2-dimensional false colour maps were generated by correlating integrated peak areas to specific positions on the measured grid. For every sample, 2-dimensional distribution maps were generated from key vibrational bands representing each individual component present in the cross section. This was undertaken in order to provide an assessment on the homogeneity of the coating and to identify regions of the surfacer that are truly representative of the composition of the layer. Single point IR spectra were extracted from these chemically uniform regions of the coating, so as to negate any chemical variability attributable to interlayer paint component migration.[19] Five replicate IR spectra were extracted from the 
primer surfacer of each sample, as replicate measurements are important in chemometric studies in order to accurately measure inter $v s$. intra-sample variation. All pre-processing and data analysis was performed via Bruker Opus software (version 7.0).

The entire spectral dataset was linearly baseline corrected and the interference fringes were removed by subtracting the appropriate sine wave from the original spectra.[19] The dataset was then mean normalised thereby eliminating any variability attributable to sample texture. Finally, the spectral data was truncated to a range of $1800-900 \mathrm{~cm}^{-1}$ as minimal variance in the spectra was observed outside of this region. Principal component analysis (PCA) $[7,9]$ was conducted on the pre-processed data, with a view to identifying any patterns between the samples as a function of the make, model and year of the vehicles.[8] All pre-processing and statistical analysis of the data was performed using the Unscrambler ${ }^{\circledR}$ X 10.2 software (CAMO Software AS, Oslo, Norway).

\section{Results and Discussion}

Synchrotron infrared imaging was utilised in the stratigraphic imaging of automotive paint cross-sections, with a view to identifying sections of the underlying layers that are chemically uniform and are thus truly representative of the composition of the coating. Figure 1e is the combined image of Figures $1 b-d$, such that the three chemical images are overlapped to produce a red-green-blue (RGB) map. RGB mapping is a form of component imaging, whereby each individual component (1b-d) is colour-coded as red, green or blue. The RGB map adds the colour portions of the selected chemical components allowing visualisation of the local distribution of all the components based upon the fundamentals of additive colour mixing. As the additive mixing of all three primary colours yields white, this region of the RGB map represents the area where all three components of the surfacer are the most abundant and therefore corresponds to the region of the coating unaffected by interlayer paint component migration. In this particular instance, it is evident that the white region in Figure 1e correlates with the mid-centre of the primer surfacer (Figure 1a). Subsequently, synchrotron FTIR spectra were extracted predominantly from the chemically uniform centre of the primer surfacer; however, this was constantly re-evaluated on a case by case basis for all the layers in each sample.

$<$ FIGURE 1 $>$ 
The sheer volume of information obtained from multivariate data makes it difficult to discern patterns and relationships. Chemometrics is a form of pattern recognition, enabling patterns to be revealed in the data, on the provision that statistically large sample populations are employed.[9] In this particular study, the sample collection contained a total of 450 spectra consisting of 75 distinct vehicles, covering a range of Australian and international manufacturers. The dataset is comprised of a diverse range of vehicles, with 13 makes and 45 different models being represented.

PCA of the primer surfacer spectral dataset revealed that $73.5 \%$ of the total variance in the data was accounted for in the first three PCs. The 3-dimensional scores plot, which is a plot of the projected samples using PC1, PC2 and PC3 as a new coordinate system, revealed that 14 distinct classes are present in the dataset and a correlation exists between the classes and the country of manufacture, the specific manufacturer and manufacturing plant (Table 1 and Figure 2). In addition to the samples classified into these 14 classes, there are samples in the dataset representing single vehicles manufactured by Nissan, Chrysler, and Jaguar. Unfortunately, the number of samples signifying these vehicles is far too small for generalisations or relationships to be deduced and as such they were excluded from the model. It is anticipated that as the dataset continues to expand, the statistical model will become more defined, consequently producing more classes corresponding to these vehicles. Interestingly, there are two distinct groupings for both the German manufactured Ford and for Hyundai. The reason for this will be discussed in detail later. As mentioned beforehand, five replicate spectra were extracted from each sample so as to assess the intra-sample variability within the surfacer layer. The differences between the replicate spectra are insignificant providing representative spectra are obtained from the chemically uniform regions of the coating; thereby ensuring compositional differences within a sample are minimal. It is important to note, similar models generated from synchrotron IR spectra extracted from the electrocoat primer and basecoat, revealed no identifiable patterns or trends. The composition of the basecoat was determined to be entirely dependent on the colour and or finish of the vehicle, which is to be expected as it is characterised by the pigments in the coat. No significant variability was observed in the IR data obtained from the electrocoat primer.

Based upon the PCA scores plot depicted in Figure 2, it is evident that 14 distinct classes are present in the spectral data. In order to ascertain which features of the spectra give rise to the discrimination between the classes, the infrared spectra of the central sample of each class was visually examined (Figure 3) and their chemical constituents were identified (Table 2). 


\section{$<$ FIGURE 2>}

\section{$<$ TABLE $1>$ \\ $<$ FIGURE 3 $>$}

The backbone of every primer surfacer was polyester; however, subtle differences were observable in the form of polyester employed (i.e. orthophthalic, isophthalic and terephthalic alkyd). Melamine was another component observed in almost all types of primer surfacers as it is commonly utilised as a cross-linking additive.[20-22] Another common constituent in the surfacer is polyurethane, which is due in part to the constructive effect it has on the dispersibility of the film in a waterborne system, whilst also producing exceptional film properties.[21-23] Epoxy resin was identifiable in over half of the surfacers as a result of the mechanical and technological features it affords the coating.[22, 23] Extenders are also widely employed in the manufacture of the primer surfacers as they affect performance parameters such as gloss, stonechip resistance and even rheology.[21] The extenders encountered in this particular study were barium sulfate, aluminium silicate and magnesium silicate. Ultimately, discrimination between classes was achieved due to distinctive permutations of the chemical components listed above, or as a result of variations in the relative abundance of components in the system (Table 2).

The factor loadings for the first three PCs are depicted in Figure 4 and were utilised to identify the spectral regions in the data responsible for the differentiation between samples on the scores plot.

\section{$<$ FIGURE 4>}

The loadings plot for PC1 revealed regions of high positive correlation at 1722, 1270 and $1251 \mathrm{~cm}^{-1}$, which are characteristic of the carbonyl and carbon-oxygen vibrational stretches attributable to the terephthalic alkyd resin.[10] Consequently, the discrimination between classes across PC1 is due to the presence or absence of terephthalic alkyd in the primer surfacer. As only the US manufactured Dodge and Jeep vehicles from class 14 contain terephthalic alkyd in the surfacer these samples attain large positive values on PC1. Similarly the remaining samples which do not contain terephthalic alkyd attain significant negative scores on PC1 and are subsequently compressed into one half of the scores plot. The loadings plot for PC2 revealed a positive correlation at $1551 \mathrm{~cm}^{-1}$, which is indicative of an in-plane vibration of the triazine ring of the melamine additive.[10] The significant negative loadings 
at $c a .1182$ and $1119 \mathrm{~cm}^{-1}$ are characteristic for barium sulfate.[10] Therefore, discrimination between samples on PC2 is achieved based upon the relative abundance of melamine and barium sulfate in the sample. The loadings plot for PC3 revealed two zones of significant negative correlation; one at approximately $1689 \mathrm{~cm}^{-1}$ which is consistent with the carbonyl stretch attributable to polyurethane, and the other at $1236 \mathrm{~cm}^{-1}$ which is the main diagnostic peak representing the isophthalic alkyd resin.[10] A broad zone of positive correlation was also observed at $1273 \mathrm{~cm}^{-1}$, which denotes the carbon-oxygen stretch attributable to the orthophthalic alkyd resin.[10] Therefore, samples are separated on PC3 based upon the presence of the orthophthalic and isophthalic alkyd resin and the abundance of polyurethane. Relating this back to the scores plot of Figure 2, it is evident that the reason why samples from class 3 attain significant positive scores on PC3 is due to the large abundance of orthophthalic alkyd and the absence of isophthalic alkyd and polyurethane in the surfacer. Conversely, the samples with large negative scores on PC3 contain a relatively large abundance of isophthalic alkyd and polyurethane with little to no orthophthalic alkyd.

It is important to note that three classes exist in the dataset representing samples obtained from General Motors manufactured Holden vehicles (class 3, 6 \& 10). It is not surprising that chemical differences in the primer surfacer are evident between the South Korean and Australian made Holden vehicles. However, a relationship was also discerned within the samples obtained from Australian factory-made Holden vehicles as a function of the year the vehicles were manufactured. In particular, samples obtained from class 3 represented vehicles manufactured post 2005, whereas samples in class 10 signify vehicles manufactured up until 2005. This relationship is of great significance as it may allow information to be acquired regarding the vehicle model, on the provision that it is known when Holden ceased production on specific vehicle lines. Furthermore, there are two Mitsubishi classes present in the dataset (class $1 \& 5$ ), with the distinction between these two groupings occurring as a function of the plant in which the vehicles were manufactured. In this particular instance, the samples from class 1 represent Mitsubishi vehicles manufactured at the Nagoya plant, whilst the samples from class 6 signify Mitsubishi vehicles produced at the Mizushima plant, thereby highlighting diversity within the manufacturer.

Interestingly, there are also two groupings in the dataset which are representative of samples obtained from the German manufactured Ford Focus (class $9 \& 13$ ). However, in this particular instance, there is no observable correlation between the two sets of classes and the common vehicle descriptors; which includes but is not limited to the model and year of the 
vehicle as well as the plant and platform used to manufacture the vehicle. Upon closer examination, a trend was discerned between the two classes and the nature and layer structure of the paint samples. Specifically, class 13 consists of Ford Focus vehicles which contain the typical 4-layered paint system, whilst the samples from class 9 represent Ford Focus vehicles which contain 6 original manufacturer coatings. As depicted in Figure 5, it is evident that the two paint systems have roughly the same total thickness, presumably to meet certain internal specifications and requirements. In this particular instance, the 4-coat system contains a much thicker primer surfacer to compensate for the fact that the 6-layered system contains an original manufacturer over-sprayed base and clear coat. The main chemical difference between the surfacers of both systems is due solely to the much higher abundance of barium sulfate present in the 4-layered system. Barium sulfate is an extender, which is used primarily to extend or stretch the primer surfacer layer further at a low cost.[23] Thus, the chemical difference between both surfacers is attributable to the fact that a large amount of barium sulfate was employed to extend the primer surfacer of the 4-layered system, so that the total thickness of the 4 and 6-layered systems are comparable. As a result, the subtle differences in the chemical composition of the two surfacers can indirectly be attributed to the number of layers in the paint system.

\section{$<$ FIGURE 5 $>$}

Additionally, there are two groupings in the dataset representing samples obtained from Hyundai manufactured vehicles. Similarly, there is no observable pattern between the two sets of classes and the typical vehicle descriptors. However, once again, a trend is observed between the chemical compositions of the surfacers from the two sets of samples as a function of the layer structure of the automotive paint samples. Specifically, the samples obtained from class 11 represent vehicles which contain an atypical 3-coat system with no original manufacturer clear coat, whilst the samples from class 12 comprise the typical 4layered scheme. As the 3-layered paint systems contain no original manufacturer clear coat the function of the primer surfacer in this particular instance is inherently more significant, as now the onus is on the primer surfacer to prevent corrosion and protect the underlying metal body. Subsequently, it is not surprising that the chemical composition of the surfacer coating varies from 3-layered and 4-layered paint systems obtained from Hyundai vehicles. It is important to note that this matrix effect was also observed in Toyota vehicles, with the 3layered Toyota paint samples obtaining a vastly different primer surfacer comparative to the 
typical 4-layered scheme. This finding ultimately reinforces the fact that different primer surfacers are designed for specific applications.[24]

\section{Conclusions}

The forensic analysis of questioned paint samples is straightforward when there is a known paint sample acquired from a suspect vehicle for comparison. It becomes inherently more challenging when there are no known samples to compare to the questioned paint sample, as is often the case when dealing with hit and run accidents. In these instances, forensic examiners rely either on the use of digital databases, such as the Paint Data Query (PDQ) database, or statistical models in order to reveal information concerning the suspect vehicle and thus procure investigative leads. In this particular instance, a chemometric model was developed using synchrotron IR data obtained from the primer surfacer. A pattern was discerned between the groupings present in the dataset as a function of the vehicle manufacturer. No discernible variation was observed in the composition of the basecoat and electrocoat primer. Previous research conducted has demonstrated that multivariate statistical analysis of IR data obtained from automotive clear coats may reveal pertinent information concerning likely vehicle origin.[15] Hence, from a forensic standpoint useful information may be acquired regarding a suspect vehicle from questioned paint samples by interrogating the clear coat, the primer surfacer or both. However, the statistical model generated from the primer surfacer offers a few advantages over the model generated from the clear coats. Firstly, the primer surfacer model affords specific information concerning the potential vehicle manufacturer, whilst the clear coat model can only provide general information regarding the origin of the vehicle. For example, the clear coat model may only provide a generalisation that the suspect vehicle was manufactured in Japan, whereas the primer surfacer model can differentiate between most of the Japanese manufacturers and thus provides specific information regarding the potential manufacturer. Furthermore, the primer surfacer being protected by the basecoat and clear coat is not as prone to weathering as the clear coats.

It is worth noting that at this point in time, only limited research has been conducted in assessing the impact that long term continuous environmental exposure has both physically and chemically on automotive clear coats. This issue may ultimately limit the applicability of statistical models generated from automotive clear coats. The advantageous feature of the primer surfacer model is that it employs chemical information which is not, or only 
minimally, impacted by deleterious effects of long-term environmental exposure. This is because the primer surfacer is sandwiched between two different coatings and as such is not directly exposed to the environment, subsequently minimising the risk of environmental degradation and thus providing an accurate representation of the composition of the coating.

The statistical model was defined from vehicles representing a range of Australian and international manufacturers. It is important to note that the international vehicles comprised vehicle models only readily exported to Australia. As a result, this statistical model can only be utilised in an Australian context. With that being said other jurisdictions could utilise the same methodology as long as statistically significant datasets were generated.

\section{Acknowledgments}

Part of this research was undertaken on the infrared microspectroscopy beamline at the Australian Synchrotron, Victoria, Australia. The authors thank Kelvin Parker and the staff of Prestige Sunroofs WA (Kardinya, Western Australia) for the automotive paint samples; Nathan Karslake and Aidon Slaney (Department of Chemistry, Curtin University) for assistance in collection of samples; Dr Paolo Raiteri (Department of Chemistry, Curtin University) for his help with data processing and the Australian Synchrotron staff for their expert help and support. Mark Maric is supported by an Australian Postgraduate Award. 


\section{Table Caption}

Table 1: The frequency of samples and the corresponding vehicles these samples represent for each class.

Table 2: Frequencies $\left(\mathrm{cm}^{-1}\right)$ for synchrotron FTIR spectra obtained from the centroid of each grouping. Note: $\mathrm{s}-$ strong, $\mathrm{m}$ - moderate, $\mathrm{w}$ - weak, $\mathrm{b}$ - broad, $\mathrm{h}$ - high, $1-$ low

\section{Figure Captions}

Figure 1: (a) Optical image of an automotive paint cross section obtained from a Mazda 3. (b) Synchrotron FTIR chemical image highlighting the distribution of polyurethane ( $c a .1690$ $\left.\mathrm{cm}^{-1}\right)$, (c) melamine (ca. $\left.1550 \mathrm{~cm}^{-1}\right)$, (d) and isophthalic alkyd (ca. $\left.1236 \mathrm{~cm}^{-1}\right)$. (e) An RGB image produced by combining and overlapping the previous three chemical images (b-d), thereby allowing visualisation of the local distribution of all three components. The polyurethane trace is depicted as red, melamine is displayed as green and isophthalic alkyd is presented as blue.

Figure 2: Two different perspectives of a 3-dimensional PCA scores plot highlighting the distribution of a population of automotive primer surfacers based upon their corresponding synchrotron IR spectra. Note classes representing vehicles, which contain only one sample were removed from the scores plot to provide clarity.

Figure 3: Synchrotron IR spectra obtained from the central sample of each class highlighting the chemical differences between the classes.

Figure 4: Factor Loadings plot of the first three PCs.

Figure 5: Synchrotron IR spectra extracted from the primer surfacer of a (a) sample from class 13 and (b) a sample from class 11. 


\section{References}

[1] A.R.W. Jackson, J.M. Jackson, Forensic Science, Pearson Prentice Hall, Essex, 2004.

[2] G. Massonnet, F. Monnard, Paint: Interpretation, in: Wiley Encyclopedia of Forensic Science, John Wiley \& Sons, Chichester, 2009.

[3] S. Bell, Forensic Chemistry, Pearson Prentice Hall, New Jersey, 2006.

[4] S. Ryland, T. Jergovich, K.P. Kirkbride, Forensic Sci. Rev., 18 (2006) 97-117.

[5] SWGMAT, Forensic Science Communications, 1 (1999).

[6] M.J. Adams, Chemometrics in analytical spectroscopy, Royal Society of Chemistry, Cambridge, 1995.

[7] R.G. Brereton, Applied chemometrics for scientists, John Wiley \& Sons Inc., Chichester, 2007.

[8] R.G. Brereton, Chemometrics: Data Analysis for the Laboratory and Chemical Plant, John Wiley \& Sons Inc., West Sussex, 2003.

[9] J.N. Miller, J.C. Miller, Statistics and Chemometrics for Analytical Chemistry, 5th ed., Pearson Education Limited, London, 2005.

[10] A. Beveridge, T. Fung, D. MacDougall, Use of infrared spectroscopy for the characterisation of paint fragments, in: B. Caddy (Ed.) Forensic Examination of Glass and Paint Analysis and Interpretation, Taylor \& Francis, London, 2001.

[11] G. Edmondstone, J. Hellman, K. Legate, G. Vardy, E. Lindsay, Can. Soc. Forensic Sci. J., 37 (2004) 147-153.

[12] G. Massonnet, W. Stoecklein, Science \& justice : journal of the Forensic Science Society, 39 (1999) 135-140.

[13] S. Ryland, G. Bishea, L. Brun-Conti, M. Eyring, B. Flanagan, T. Jergovich, D. MacDougall, E. Suzuki, J. Forensic Sci., 46 (2001) 31-45.

[14] J. Zieba-Palus, J. Mol. Struct., 512 (1999) 327-335.

[15] M. Maric, W. van Bronswijk, S.W. Lewis, K. Pitts, Anal. Methods, 4 (2012) 26872693.

[16] G. Sauzier, M. Maric, W. van Bronswijk, S.W. Lewis, Anal. Methods, (2013) DOI: 10.1039/C3AY40458G.

[17] K. Flynn, R. O'Leary, C. Lennard, C. Roux, B.J. Reedy, J. Forensic Sci., 50 (2005) 832-841.

[18] L.M. Miller, R.J. Smith, Vib. Spectrosc., 38 (2005) 237-240.

[19] M. Maric, W. van Bronswijk, S.W. Lewis, K. Pitts, D.E. Martin, Forensic Sci. Int., 228 (2013) 165-169.

[20] J. Bentley, Composition, manufacture and use of paint, in: B. Caddy (Ed.) Forensic Examination of Glass and Paint Analysis and Interpretation, Taylor \& Francis, London, 2001 .

[21] H. Streitberger, K. Dossel, in, Wiley-VCH, Weinheim, 2008.

[22] P. Nanetti, Coatings from A to Z, Vincentz Network, Hannover, 2006.

[23] U. Poth, Automotive Coatings Formulation: European Coatings Tech Files, Vincentz Network, Hannover, 2008.

[24] D.W. Parks, D.H. Jacobs, How to Paint Your Car, Motorbooks International, 2013. 
Figure 1:

\section{Low}

High

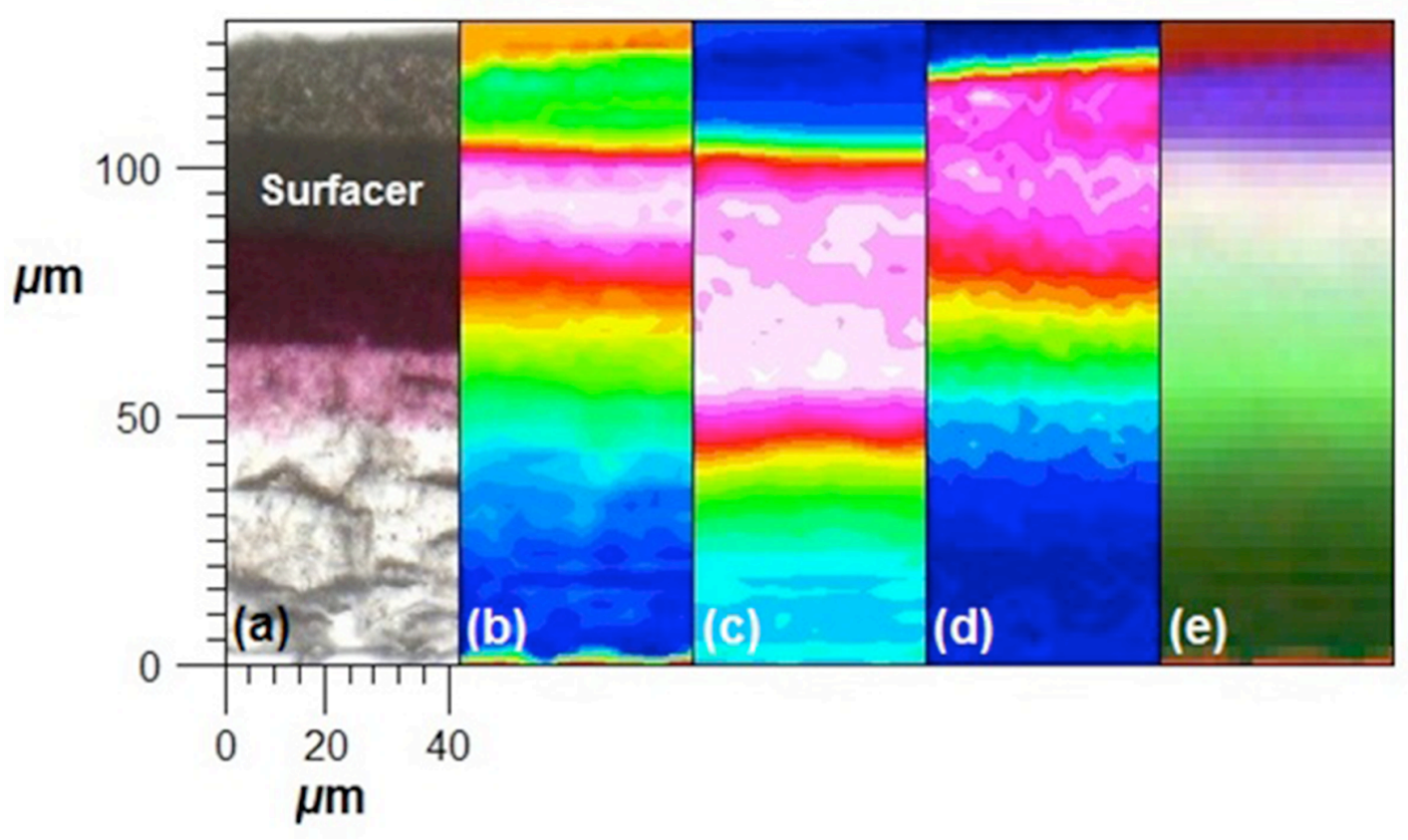

Figure 2:
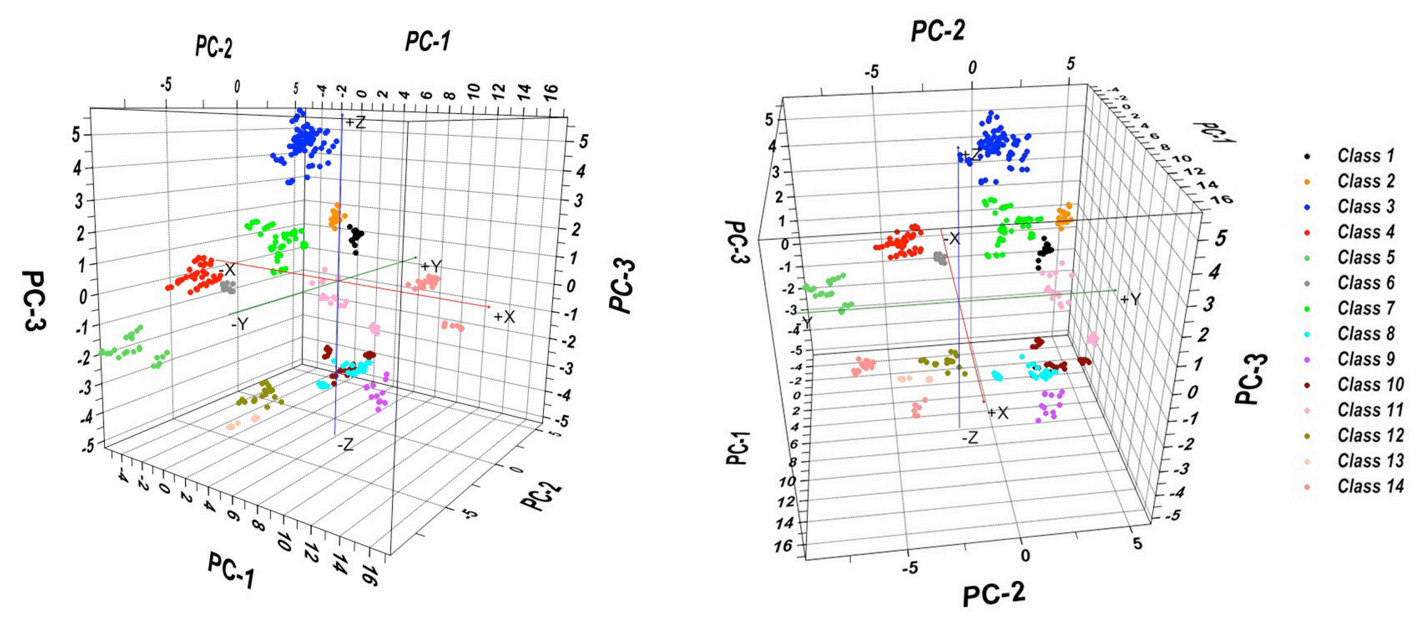
Figure 3:

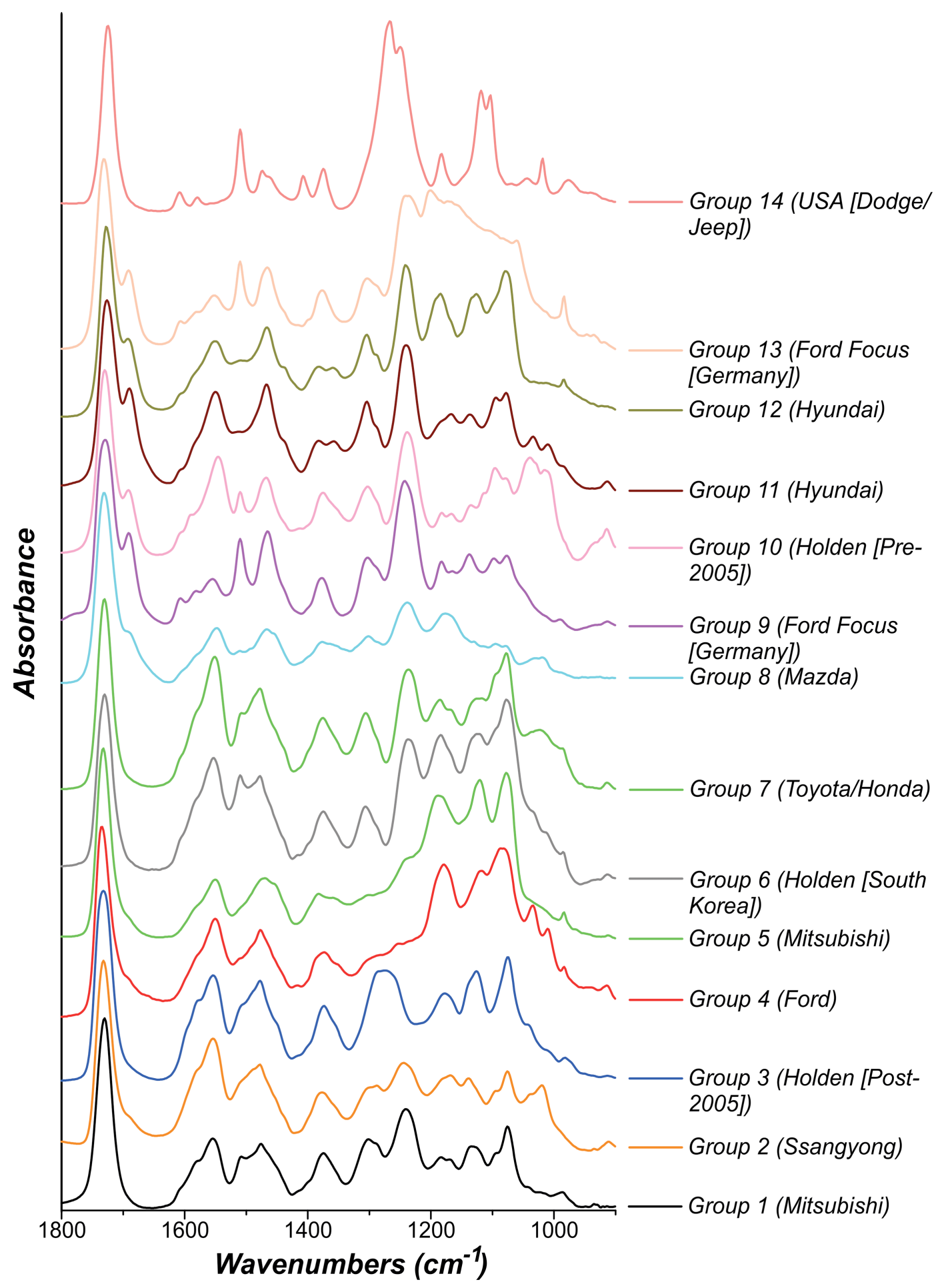


Figure 4:

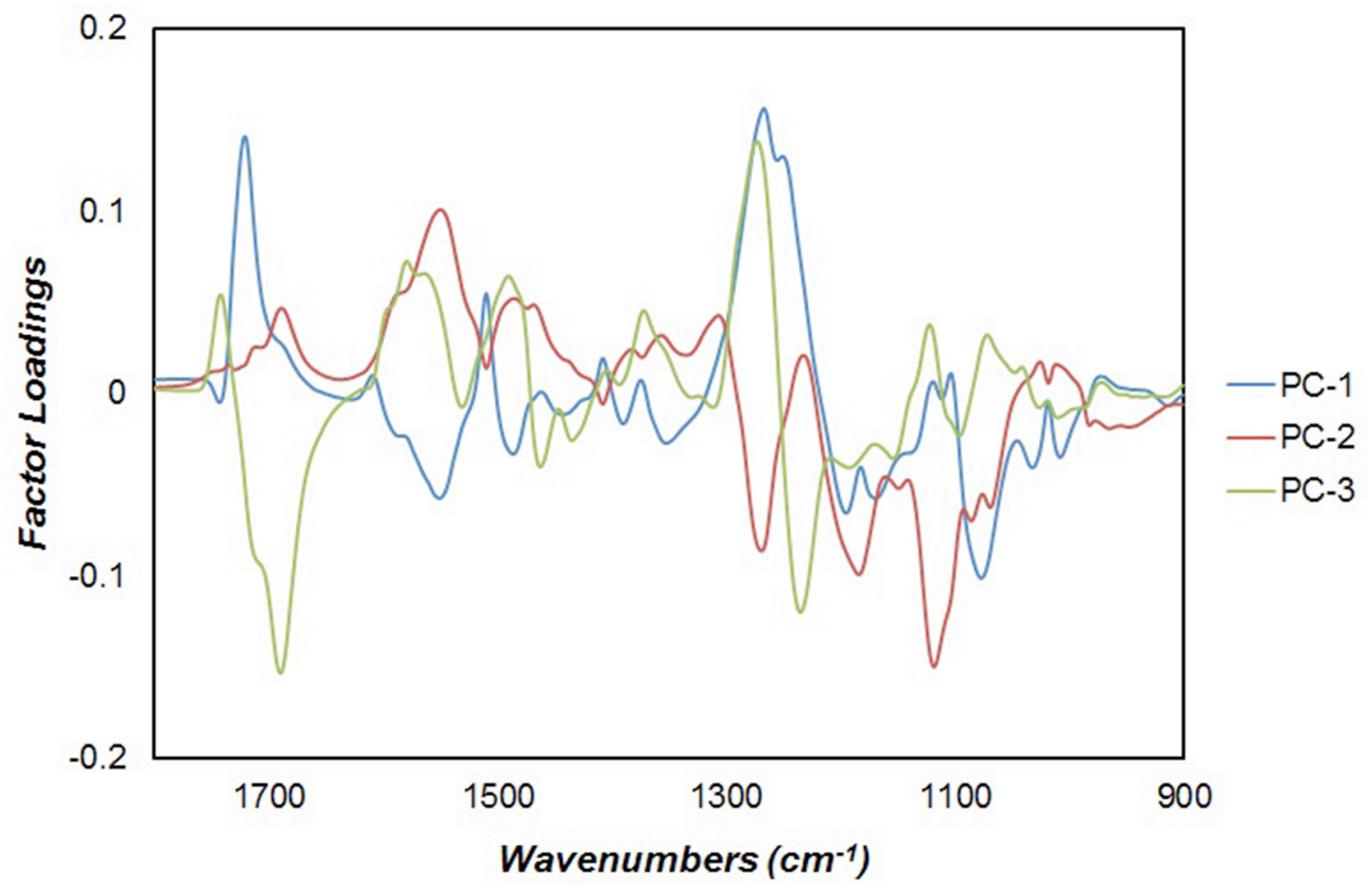

Figure 5:

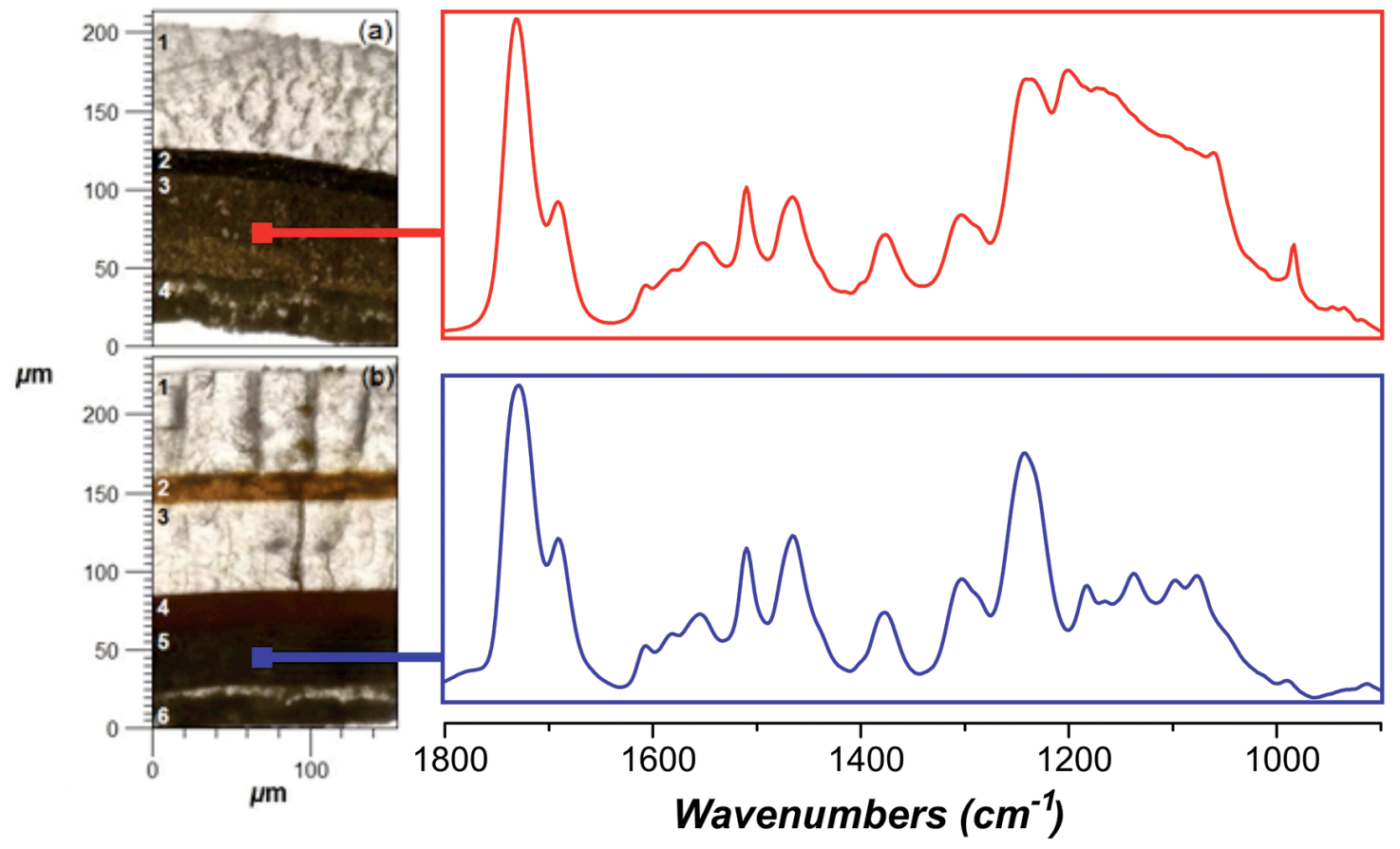


Table 1: Classes revealed by PCA of primer surfacer spectral dataset

\begin{tabular}{|c|c|c|}
\hline Class & Vehicle/s represented & No. of samples \\
\hline 1 & Mitsubishi (Nagoya) & 3 \\
\hline 2 & SsangYong & 3 \\
\hline 3 & Holden post-2005 (Australia) & 15 \\
\hline 4 & Ford (Australia) & 10 \\
\hline 5 & Mitsubishi (Mizushima) & 4 \\
\hline 6 & Holden (South Korea) & 3 \\
\hline 7 & Toyota/Honda & 10 \\
\hline 8 & Mazda & 5 \\
\hline 9 & Ford (Germany) & 2 \\
\hline 10 & Holden pre-2005 (Australia) & 5 \\
\hline 11 & Hyundai & 5 \\
\hline 12 & Hyundai & 4 \\
\hline 13 & Ford (Germany) & 2 \\
\hline 14 & Dodge/Jeep (US) & 4 \\
\hline
\end{tabular}




\begin{tabular}{|c|c|c|}
\hline Class & Infrared Absorption Frequencies $\left(\mathrm{cm}^{-1}\right)$ & Composition \\
\hline 1 (Mitsubishi [Nagoya]) & $\begin{array}{l}1731(\mathrm{~s}), 1554(\mathrm{~m}), 1508(\mathrm{~m}), 1476(\mathrm{~m}), 1375(\mathrm{~m}), 1301(\mathrm{~m}), 1242(\mathrm{~m}), 1183(\mathrm{~m}), 1135 \\
(\mathrm{~m}), 1076(\mathrm{~m}), 986(\mathrm{w})\end{array}$ & $\begin{array}{c}\text { Isophthalic alkyd (m), Melamine (m), Epoxy (m), } \\
\text { Barium sulfate }(\mathrm{m})\end{array}$ \\
\hline 2 (SsangYong) & $\begin{array}{l}1731(\mathrm{~s}), 1553(\mathrm{~m}), 1478(\mathrm{~m}), 1376(\mathrm{~m}), 1289(\mathrm{~m}), 1245(\mathrm{~m}), 1168(\mathrm{~m}), 1139(\mathrm{~m}), 1076 \\
(\mathrm{~m}), 1020(\mathrm{~m}), 912(\mathrm{w})\end{array}$ & $\begin{array}{l}\text { Isophthalic alkyd (m), Melamine (m), Magnesium } \\
\text { silicate }(\mathrm{m})\end{array}$ \\
\hline 3 (Holden | & $\begin{array}{l}1732(\mathrm{~s}), 1554(\mathrm{~m}), 1478(\mathrm{~m}), 1374(\mathrm{~m}), 1272(\mathrm{bm}), 1177(\mathrm{~m}), 1126(\mathrm{~m}), 1075(\mathrm{~s}), 983 \\
(\mathrm{w})\end{array}$ & $\begin{array}{l}\text { Orthophthalic alkyd }(\mathrm{m}), \text { Melamine }(\mathrm{m}) \text {, Barium } \\
\text { sulfate }(\mathrm{m})\end{array}$ \\
\hline 4 (Ford) & $\begin{array}{l}1735(\mathrm{~s}), 1550(\mathrm{~m}), 1477(\mathrm{~m}), 1374(\mathrm{~m}), 1238(\mathrm{~m}), 1179(\mathrm{~m}), 1114(\mathrm{~m}), 1086(\mathrm{~s}), 1035 \\
(\mathrm{~m}), 1010(\mathrm{~m}), 984(\mathrm{~m}), 914(\mathrm{w})\end{array}$ & $\begin{array}{l}\text { Isophthalic alkyd (m), Melamine }(\mathrm{m}), \text { Barium } \\
\text { sulfate }(\mathrm{m}) \text {, Aluminium silicate }(\mathrm{m})\end{array}$ \\
\hline $\begin{array}{l}5 \text { (Mitsubishi } \\
{[\text { Mizushima]) }}\end{array}$ & $\begin{array}{l}1732(\mathrm{~s}), 1551(\mathrm{w}), 1469(\mathrm{w}), 1383(\mathrm{w}), 1301(\mathrm{w}), 1236(\mathrm{~m}), 1184(\mathrm{~s}), 1120(\mathrm{~s}), 1074(\mathrm{~s}), \\
984(\mathrm{w})\end{array}$ & Isophthalic alkyd (m), Melamine (1), Barium sulfate \\
\hline 6 (Holden [South Korea]) & $\begin{array}{l}1730(\mathrm{~s}), 1554(\mathrm{~m}), 1510(\mathrm{~m}), 1478(\mathrm{~m}), 1375(\mathrm{~m}), 1306(\mathrm{~m}), 1237(\mathrm{~s}), 1184(\mathrm{~s}), 1125(\mathrm{~s}) \\
1076(\mathrm{~s}), 1036(\mathrm{~m}), 1013(\mathrm{~m}), 986(\mathrm{w}), 914(\mathrm{w})\end{array}$ & $\begin{array}{l}\text { Isophthalic alkyd }(\mathrm{m}), \text { Melamine }(\mathrm{m}) \text {, Epoxy }(\mathrm{m}) \\
\text { Barium sulfate }(\mathrm{h}) \text {, Aluminium silicate }(\mathrm{m})\end{array}$ \\
\hline 7 (Toyota & $\begin{array}{l}1730(\mathrm{~s}), 1551(\mathrm{~s}), 1508(\mathrm{~m}), 1478(\mathrm{~m}), 1376(\mathrm{~m}), 1305(\mathrm{~m}), 1237(\mathrm{~s}), 1182(\mathrm{~m}), 1171 \\
(\mathrm{~m}), 1130(\mathrm{~m}), 1079(\mathrm{~s}), 985(\mathrm{w})\end{array}$ & $\begin{array}{l}\text { Isophthalic alkyd }(\mathrm{m}), \text { Melamine }(\mathrm{h}), \text { Epoxy }(\mathrm{m}) \text {, } \\
\text { Barium sulfate }(\mathrm{m})\end{array}$ \\
\hline 8 [Mazda] & $\begin{array}{l}1731(\mathrm{~s}), 1691(\mathrm{w}), 1548(\mathrm{w}), 1511(\mathrm{w}), 1467(\mathrm{w}), 1377(\mathrm{w}), 1302(\mathrm{w}), 1239(\mathrm{~m}), 1176 \\
(\mathrm{~m}), 1095(\mathrm{w}), 1078(\mathrm{w}), 1031(\mathrm{w}), 1019(\mathrm{w})\end{array}$ & $\begin{array}{l}\text { Isophthalic alkyd (m), Melamine (1), Polyurethane } \\
\text { (1), Epoxy (1), Aluminium silicate (1) }\end{array}$ \\
\hline 9 (Ford [Germany]) & $\begin{array}{l}1729(\mathrm{~s}), 1691(\mathrm{~m}), 1607(\mathrm{w}), 1581(\mathrm{w}), 1553(\mathrm{w}), 1510(\mathrm{~m}), 1466(\mathrm{~m}), 1377(\mathrm{w}), 1303 \\
(\mathrm{~m}), 1243(\mathrm{~s}), 1182(\mathrm{~m}), 1138(\mathrm{~m}), 1098(\mathrm{~m}), 1077(\mathrm{~m}), 915(\mathrm{w})\end{array}$ & $\begin{array}{l}\text { Isophthalic alkyd (m), Melamine (1), Polyurethane } \\
\qquad(\mathrm{m}), \text { Epoxy }(\mathrm{m})\end{array}$ \\
\hline 10 [Holden (Pre-2005)] & $\begin{array}{l}1730(\mathrm{~s}), 1691(\mathrm{~m}), 1546(\mathrm{~s}), 1510(\mathrm{~m}), 1468(\mathrm{~m}), 1376(\mathrm{~m}), 1303(\mathrm{~m}), 1238(\mathrm{~s}), 1182 \\
(\mathrm{~m}), 1166(\mathrm{~m}), 1136(\mathrm{~m}), 1095(\mathrm{~m}), 1078(\mathrm{~m}), 1034(\mathrm{~s}), 1012(\mathrm{~m}), 915(\mathrm{~m})\end{array}$ & $\begin{array}{l}\text { Isophthalic alkyd (m), Melamine (h), Polyurethane } \\
(\mathrm{m}) \text {, Epoxy (m), Aluminium silicate }(\mathrm{h})\end{array}$ \\
\hline 11 (Hyundai) & $\begin{array}{l}1725(\mathrm{~s}), 1690(\mathrm{~m}), 1552(\mathrm{~m}), 1468(\mathrm{~m}), 1382(\mathrm{w}), 1358(\mathrm{w}), 1304(\mathrm{~m}), 1240(\mathrm{~s}), 1165 \\
(\mathrm{w}), 1138(\mathrm{w}), 1096(\mathrm{~m}), 1078(\mathrm{~m}), 1035(\mathrm{w}), 1010(\mathrm{w}), 915(\mathrm{w})\end{array}$ & $\begin{array}{l}\text { Isophthalic alkyd (m), Melamine (m), Polyurethane } \\
\qquad(\mathrm{m}) \text {, Aluminium silicate }(\mathrm{l})\end{array}$ \\
\hline 12 (Hyundai) & $\begin{array}{l}1725(\mathrm{~s}), 1692(\mathrm{~m}), 1551(\mathrm{~m}), 1509(\mathrm{~m}), 1466(\mathrm{~m}), 1382(\mathrm{~m}), 1359(\mathrm{~m}), 1304(\mathrm{~m}), 1240 \\
(\mathrm{~s}), 1184(\mathrm{~s}), 1124(\mathrm{~s}), 1079(\mathrm{~s}), 984(\mathrm{w}), 915(\mathrm{w})\end{array}$ & $\begin{array}{l}\text { Isophthalic alkyd (m), Melamine (m), Polyurethane } \\
(\mathrm{m}) \text {, Epoxy (m), Barium Sulfate }(\mathrm{m})\end{array}$ \\
\hline 13 (Ford [Germany]) & $\begin{array}{l}1731(\mathrm{~s}), 1691(\mathrm{~m}), 1607(\mathrm{w}), 1580(\mathrm{w}), 1551(\mathrm{w}), 1510(\mathrm{~m}), 1466(\mathrm{~m}), 1377(\mathrm{w}), 1303 \\
(\mathrm{~m}), 1240(\mathrm{~s}), 1201(\mathrm{~s}), 1170(\mathrm{~s}), 1061(\mathrm{~m}), 984(\mathrm{w})\end{array}$ & $\begin{array}{l}\text { Isophthalic alkyd (m), Melamine (1), Polyurethane } \\
\qquad(\mathrm{m}) \text {, Epoxy }(\mathrm{m}) \text {, Barium sulfate }(\mathrm{h})\end{array}$ \\
\hline 14 (USA [Dodge/Jeep]) & $\begin{array}{l}1724(\mathrm{~s}), 1608(\mathrm{w}), 1580(\mathrm{w}), 1510(\mathrm{~m}), 1474(\mathrm{w}), 1408(\mathrm{w}), 1375(\mathrm{w}), 1269(\mathrm{~s}), 1249(\mathrm{~s}), \\
1183(\mathrm{w}), 1120(\mathrm{~m}), 1105(\mathrm{~m}), 1044(\mathrm{w}), 1019(\mathrm{w}), 976(\mathrm{w})\end{array}$ & Terephthalic alkyd (h), Epoxy (m) \\
\hline
\end{tabular}

Table 2: Frequencies $\left(\mathrm{cm}^{-1}\right)$ for synchrotron FTIR spectra obtained from the centroid of each grouping. Note: $\mathrm{s}-\mathrm{strong}, \mathrm{m}-\mathrm{moderate}, \mathrm{w}-\mathrm{weak}, \mathrm{b}-\mathrm{broad}$, h - high, 1 - low. 
